\title{
Buccal Fat Pad- Stromal Vascular Fraction as a New Regenerative Source in Treatment of Maxillofacial Bone Defects: A Case Series Study
}

\author{
Mahboubeh Bohlouli \\ Shahid Beheshti University \\ Farshid Bastami \\ Shahid Beheshti University \\ Nasser Nadjmi \\ University of Antwerp \\ Hanieh Nokhbatolfoghahei \\ Shahid Beheshti University \\ Arash Khojasteh ( $\nabla$ arashkhojasteh@sbmu.ac.ir) \\ Shahid Beheshti University
}

\section{Research Article}

Keywords: Buccal fat pad, stromal vascular fraction, bone regeneration, maxillofacial defect, stem cell

Posted Date: May 3rd, 2021

DOI: https://doi.org/10.21203/rs.3.rs-441758/v1

License: (a) This work is licensed under a Creative Commons Attribution 4.0 International License. Read Full License 


\section{Abstract}

The study aimed to evaluate the biological properties of the buccal fat pad (BPF) derived tissue stromal vascular fractions (tSVF) using a mechanical device (Minceolip ${ }^{\circledR}$ ), compared to BFP- cellular SVF (cSVF) in vitro. Also, the efficiency, safety, and feasibility of the BFP-tSVF were assessed in patients with various maxillofacial defects. Isolation of CSVF and-tSVF from BFP was performed through enzymatic and Mechanical devices. They were evaluated in terms of cell counting and viability, expression surface markers, kinetic growth, and tri-lineage differentiation. Ten patients with jaw bone defects received the BFP- tSVF along with an absorbable gelatin sponge in the clinical stage. Effectiveness and safety were measured by computed tomography. The BFP- tSVF showed high cell viability and cell surface markers, specifically CD45, CD31, and CD34. There was no difference in their kinetic growth and multi-lineage differentiation potential between enzymatic and mechanical approaches. Bone regeneration was significantly enhanced in the patients who received BFP- tSVF than control groups in both segmental $(33.50 \% \pm 4.95$ vs. $5.00 \% \pm 7.07$, P-value: 0.042$)$ and partial resection $(89.33 \% \pm 2.08$ vs. $10.33 \% \pm 10.02$, P-value: 0.000$)$. There were no intervention-related adverse events reported in the patients. These results confirmed that the BFP- tSVF is effective, safe, and feasible for maxillofacial bone defects patients. It will be necessary to evaluate of clinical efficiency of BFP- tSVF for improving bone regeneration in future studies.

\section{Introduction}

Stromal vascular fraction (SVF) is used in a broad spectrum of various medical conditions, from threatening life (i.e., cardiomyopathy, pulmonary fibrosis) to less-lethal states (i.e., chronic wound, osteoarthritis) ${ }^{1-5}$. This fraction consists of heterogeneous cellular populations, such as mesenchymal progenitors, endothelial progenitors, supra-adventitial adipose stromal cells, and pericytes, which have essential roles in angiogenesis, anti-inflammatory, and anti-fibrosis ${ }^{6}$. SVF is classified into cellular-derived SVF (cSVF) and tissue-derived SVF (tSVF) ${ }^{7}$ according to preparation procedure, including enzymatic and mechanical, respectively.

In the enzymatic harvesting procedure, cells' phenotypical and functional properties alter via the degradation of cell surface and extracellular matrix (ECM) proteins. Therefore, their cells' number and quality depend on isolation methodology, equipment, chemical reagents, and protease activity of each batch of enzymes ${ }^{7}$. The translation of the enzymatic digestion procedure into clinical needs to get the manufacturing grade (GMP) standards for operating protocols from food and drug administration (FDA) agencies ${ }^{8}$.

Recently, tSVF has attracted many scientists' attention by preserving tissue native microenvironment and original relevant characteristics. The novel devices were patented to improve the efficiency and feasibility of tSVF isolation according to three physical methods, including centrifugation, filtration, and gravity sedimentation ${ }^{9-13}$. Notably, the mechanical procedure is considered a "minimal manipulated approach" in the US and does not need to be approved by the FDA agencies ${ }^{14}$.

Buccal Fat Pad (BFP) is a primary adipose tissue source between the buccinator muscle and other superficial muscles, containing abundant neural-crest-derived stem cells and vascular supply ${ }^{15-20}$. This tissue has two main advantages: easy harvesting with minimal donor site morbidity and complication rate and also having 
the same size in all people, independent of fat distribution, body weight, and age ${ }^{16,21}$. Our previous experimental study indicated that BFP derived stem cells (BFPdSCs) had a higher proliferation rate, osteogenesis, and the expression of angiogenic surface cell markers compared to isolated stem cells from other adipose tissues, i.e., abdomen and hip ${ }^{21}$.

On the other hand, one of the main challenges in regenerative medicine is a reconstruction of critical-sized jaw bone defects, and either large span defect originated from an odontogenic tumor resection or vertical agerelated jaw atrophy. As a traditional standard treatment technique, autologous bone grafting is associated with several disadvantages, such as infection, donor site morbidity, high cost, and prolonged hospitalization ${ }^{19}$. With the current advances in cell research, cell-based regenerative medicine approaches move from the bench to the patients' bedside to restore these defects to functional capability and appropriate esthetic form. In recent years, the use of intact BFP or BFPdSCs has been suggested to improve the bone regeneration in maxillomandibular atrophy, post-excision defects, alveolar cleft defect augmentation of the maxillary sinus floor ${ }^{17-20}$. However, the application of BFP- tSVF to improve jaw bone regeneration is a main challenge in regenerative medicine.

In the current study, a novel mechanical device, called Minceolip ${ }^{\circledR}$, was fabricated to isolating tSVF from intact BFP during surgery. The biological properties of these cellular properties, such as cell viability, cell surface markers, kinetic growth, and multi-differentiation potential, evaluate and compare with BFP- cSVF. Moreover, the effectiveness of mechanical tSVF harvesting from a BFP local niche for promotion jaw bone regeneration assesses.

\section{Results}

\subsection{BFP- tSVF are a heterogeneous vital cell population that have all stemness properties}

The nucleated cell yield was $581.00 \pm 20.075 \times 10^{3}$ per milliliter of BFP- tSVF, isolating using the Minceolip ${ }^{\circledR}$ device. Annexin-PI staining evaluated the percentage of viable nucleated cells after isolation of BFP- tSVF. These flow cytometry results demonstrated that $93.7 \% \pm 0.64$ of these cells were viable (Fig. 1(A, B)). Moreover, According to the guidelines of the international society for cellular therapy (ISCT) and the international federation for adipose therapeutics and sciences (IFATS) ${ }^{22}$, the percentage of cell positive for progenitor (CD34) and hematopoietic (CD45 and CD14), mesenchymal stem cell (CD90 and CD44), and vascular (CD31) markers were $66.5 \pm 0.62,79.16 \pm 1.23,80.95 \pm 1.6,26.40 \pm 1.9,89.63 \pm 1.75$, and $77.37 \pm 2.4$ (Fig. 1C).

To evaluate the stemness properties of BFP-tSVF, including expansion rate, PDT, and multi-lineage differentiation, were analyzed and compared with isolated cSVF by ENZ protocol. Seven-day after cell culture, the morphology of isolated cells by both protocols was revealed that these cells had a spinal fibroblast-like shape with spiral growth. Also, the growth trend showed that the number of isolated cells by the mechanical protocols was significantly lower than in another group. Other differences between the two groups were the presence of tSVF fragments in the plate culture, and fibroblast-like cells were migrating from them outward (Fig. 2 (A-B)). 
The expansion rate in mechanical and ENZ groups was associated with a steady increase from PN2 to PN9 ( 39.67 and 32.84 fold, respectively). Although the mechanical protocol in isolated cells was higher than the ENZ technique in all PN, this difference was no statistically significant (Fig. 2C).

PDT increased in both groups during the time so that a statistically significant seen on 30 and 35 days compared to 5 days in intragroup comparison (P-value $<0.0001)$. Also, this time was significantly lower $(\mathrm{P}$ value $<0.0001)$ in the mechanical group compared to the ENZ group at 30 days $(5.02 \pm 1.08$ and $8.16 \pm 1.25$, respectively) and 35 days $(8.9 \pm 0.48$ and $15.05 \pm 0.58$, respectively) (Table 1$)$.

Table 1

Population doubling time (days) of isolated cells through ENZ and mechanical protocol.

\begin{tabular}{|lll|}
\hline Time & ENZ & Mechanical \\
\hline $\mathbf{5}$ & $1.46 \pm 0.74$ & $2.06 \pm 0.69$ \\
\hline $\mathbf{1 0}$ & $2.01 \pm 0.78$ & $1.92 \pm 0.11$ \\
\hline $\mathbf{1 5}$ & $2.61 \pm 0.16$ & $2.56 \pm 0.11$ \\
\hline $\mathbf{2 0}$ & $2.31 \pm 0.22$ & $2.19 \pm 0.10$ \\
\hline $\mathbf{2 5}$ & $3.00 \pm 0.40$ & $2.55 \pm 0.52$ \\
\hline $\mathbf{3 0}$ & $8.16 \pm 1.25^{\star}$ & $5.02 \pm 1.08^{\star}$ \\
\hline $\mathbf{3 5}$ & $15.05 \pm 0.58^{\#}$ & $8.9 \pm 0.48^{\#}$ \\
\hline ENZ: Enzymatic protocol. ${ }^{* \#} \mathrm{p}$ Value $<0.0001$ \\
\hline
\end{tabular}

Tri-lineage differentiation was evaluated by standard culture-differentiating conditions in vitro to compare the multipotency of cells isolated from mechanical and ENZ protocols. Osteogenic induction of the cells resulted in the morphological transition of fibroblast-like cells into branched morphology [31] and the presence of calcium deposits in ECM. Also, inductive adipogenesis and chondrogenic medium lead to lipid formation accumulated in the cytoplasm and proteoglycan, respectively, in both groups (Fig. 3A). Semi-quantitative analysis of these staining demonstrated that there was no statistically significant difference in multidifferentiation potential between two groups ( $P$-value $=0.82,0.52$, and 0.64 for Alizarin Red, Oil Rel 0 , and Toluidine Blue, respectively) (Fig. 3B)

\subsection{BFP- tSVF promote bone regeneration in jaw bone regeneration}

The evaluation of the adverse events' occurrence demonstrated that nine patients had uneventful healing, without any unexpected pain, signs of local inflammation, infection, and wound dehiscence. All patients showed considerable chewing and speech enhancement after six months. Desirable outcomes in terms of the esthetic and functional were also observed in all patients in a year follow-up. There was no report from loosening in screws or a history of jaw dislocation. However, the oral inflammatory exudates from the surgical site and pain were observed eight months postoperatively in a patient that was received BFP-tSVF based therapy based on segmental resection. The removal of a titanium reconstruction plate was performed to 
decrease inflammation for the patient. Thus, her pain and inflammatory signs have been removed after the treatment, and favorable bone healing was observed after one year.

In the segmental resection group, the new bone formation analysis showed a significant high (P-value: 0.042) in patients who received BFP-tSVF (33.50 \pm 4.95$)$ compared to the control group (5.00 \pm 7.07$)$ (Fig. 4). Although all patients need autologous bone grafting from iliac crest bone after one year. This amount of autologous bone will be much lower for patients treated with BFP- tSVF. The radiographic analysis demonstrated that bone regeneration was statistically enhanced in BFP-tSVF $(89.33 \pm 2.08)$ than the control group (10.33 \pm 10.02$)$ in the marginal resection techniques (P-value: 0.000$)$ (Fig. 5). Also, these patients did not require autologous bone grafting.

\section{Discussion}

This study's main outcome was evaluating the biological properties and efficiency of mechanical derived BFPtSVF. Moreover, we aimed to assess the safety and feasibility of BFP- tSVF, as a one-step surgical procedure, in a clinical condition for patients with maxillofacial defects.

The cell counting data indicated that BFP- tSVF isolation by Miniceolip® device extracted more cells than inter-syringe and vortexing/centrifugation techniques that used lipoaspirate tissue ${ }^{23,24}$. This result was approximately similar to the number of isolated cells by enzymatic protocols 24,25 . Assess the viability of BFPSVF by annexin-PI staining demonstrated that a high percentage of them $(93.7 \pm 0.64)$ were viable cells, similar to enzymatic-isolated SVF $\left(90.77 \pm 2.73^{24}\right.$ and $\left.94-95^{26}\right)$. These results were contrary to the study of Benoit Chaput et al. that the viability of SVF extracted by two mechanical techniques (Vortexing/centrifugation and Dissociation by inter-syringe) was significantly lower ( $54.53 \% \pm 7.55$ and $45.53 \% \pm 3.49$, respectively) compared to an enzymatic method $(90.77 \% \pm 2.73)^{24}$. While in another study, simultaneous use of mechanical devices, including Nanofat and LipocubeNano, and enzymatic protocol led to an increase in the number of isolated CSVF $(1.44 \times 106$ and $2.24 \times 106$, respectively) and their viability $(96.5 \%)^{27}$. One of the reasons for this difference in the results is the harvesting area of adipose tissue, abdominal or hip tissues compared to BFP in our study, affecting the fat grafts' quality ${ }^{26}$. The presence of many blood vessels and embryonic stem cells ${ }^{15,28,29}$ in the BFP tissue may lead to increased cell viability than the abdominal and hip tissues used. Also, the lack of mechanical and low osmolality stress during intact BFP tissue harvesting may enhance cell viability compared to liposuction. The number of cellular components that have been wasted during washing of the suctioned adipose tissue may decrease the regenerative capacity of tSVF 30,31 .

Phenotypic evaluation of the BFP- tSVF demonstrated a heterogeneous population of stem/ progenitor cells (such as stromal, endothelial, and hematopoietic) and blood lineage cells, specifically macrophages. Interestingly, the percentage of CD $45^{+}$cells $(79.16 \pm 1.23 \%)$, as a common leukocyte antigen, was significantly high than other studies $(3.1-30.3 \%)^{16,32-34}$. This difference is justifiable because the CD45 expression reflects the vascularization level in adipose tissue ${ }^{32}$. In this regard, the percentage of endothelial progenitor/ cell marker (CD31) in BFP-tSVF demonstrated an increase from than extracted SVF in other studies (77.37 \pm $2.4 \%$ vs. $1.8-26.7 \%)^{32-35}$. Moreover, this result was similar to the percentage of CD31 + cells in bone marrow 
aspirate and peripheral blood ( $97.6 \%{ }^{36}$ and $70-78 \%{ }^{37}$, respectively). On the other hand, the percentage of CD34 ${ }^{+}$cells was higher in BFP-tSVF $(66.5 \% \pm 0.62)$ compared to Nanofat processing devices (LipocubeNano: $18.84 \%$, and Nanofat: $7.9 \%{ }^{27}$. The evaluation of cSVF revealed a significant difference in the percentage of $\mathrm{CD}_{34}{ }^{+}$cells resulting from various concentrations of enzyme, tissue type, and isolation procedure ${ }^{16,32-34}$. Overall, Given that several studies, CD31 + and CD34 + cells enhance bone regeneration through angiovascularization, osteogenesis, and immunomodulation stimulus; therefore we expect to see these results in the clinical evaluation ${ }^{37-39}$.

To evaluate the kinetic growth of BFP-tSVF using the Minceolip ${ }^{\circledR}$ device, morphology, expansion rate, and PDT were compared CSVF in vitro. The tSVF- derived cells exhibited a nearly identical expansion rate with stromal cSVF- derived cells in the present study. Contrary to the results, Benoit Chaput et al. reported that the enzymatic method's expansion rate was significantly high compared to mechanical methods, including vortexing and centrifugation and dissociation by intersyringe processing ${ }^{24}$. Moreover, the shortest doubling time was beholden in the stromal cells isolated by the Minceolip ${ }^{\circledR}$ device than ENZ protocol after 30 days. Considering that increasing doubling time is one of the signs of cell aging ${ }^{40}$, it can be concluded that isolated stromal cells by enzymatic method enter the aging phase earlier. However, these results need further study in the future.

Adipose-derived stromal cells are introduced as multipotent cells with differentiation potential into mesenchymal and non-mesenchymal origin cell types ${ }^{24,31}$. Hence, our results showed that Minceolip ${ }^{\circledR}$ derived stromal cells had tri-lineage differentiation potential, including adipose, osteoblast, and chondrocyte, similar to ENZ protocol. These results were consistent with several studies that used other mechanical protocols to isolate tSVF 24,27 .

Given the promising results in the experimental phase about BFP- tSVF, efficiency, safety, and feasibility of the cellular product evaluated to promote bone regeneration in the patients with critical-sized jaw bone defects, including vertical age-related jaw atrophy and large span defect originated from odontogenic tumor resection.

Computed tomographic evaluation of the patients following surgery demonstrated new regenerate bone in the respected segmental patients who received BFP- tSVF ( 6.7 fold) and in marginal respected patients ( 8.6 fold) resection compared to control groups. In the respected segmental defect, this amount of bone formation during routine one year follow up reducing the amount of autologous iliac bone in a second surgery and can lead to a decrease in adverse events, such as pain, donor site morbidity, hospitalization, and cost, and increase patients' satisfaction. This advantage was more pronounced in marginal respected patients who received BFP- tSVF than the control group. Extraoral bone harvesting was eliminated in these patients. The patients may need bone augmentation from Intralock oral donor sites or using bone substitutes. These results were consistent with other clinical studies that indicated adipose-derived stem cells and fresh cSVF could enhance new bone regeneration in the different bone defects $1,19,41,42$.

In safety evaluation, a chronic inflammatory reaction in one patient received BFP-tSVF that was resolved by removing the titanium reconstruction plate related to the allergic reaction to Ti particles, activating macrophages, and increasing TNF- $a^{43,44}$. Hence, the safety parameters demonstrated that no related adverse 
events occurred during follow-up. This result agrees with our previous clinical studies that used nonmanipulated BFP as a membrane for covering the bone graft, had no adverse events in sinus augmentation 17 and the atrophied edentulous maxilla ${ }^{18}$.

The presented mechanical protocol in this clinical study by the Minceolip® device is feasible to isolate BFPtSVF. This adipose tissue can harvest through local anesthesia with minimal adverse events for patients and cost in a short time. The preparation time for isolation SVF by the Minceolip device (10 min) was lower than the enzymatic method (80min). This mechanical procedure is a minimal manipulation procedure according to regulations of HCT/P (human cell, tissue, and cellular and tissue-based product) under Sect. 361 of the public health service ACT authorizes FDA ${ }^{14}$. It can be done at the operating theatre's surgical table, decreasing the cost and total time of regenerative technique compared to other enzymatic or lab process procedures. It is an enzymatic-free technique that does not alter the biological native of the tissue.

\section{Conclusion}

The experimental results suggested that the mechanical derived BFP-tSVF by delivering stromal and vascular cells may increase the surgical defects' regenerative capacity during the healing period. More studies with a larger sample size need to be more conclusive for the effectiveness of the minimal manipulation procedure in Jaw bone regeneration.

\section{Material And Methods}

\subsection{Ethical approval of the clinical study}

The clinical study was performed according to the World Medical Association Declaration of Helsinki's declared ethical principle. All clinical study protocols were submitted and approved by the Ethical Committee of the Shahid Beheshti University of Medical Sciences (Approval number IR.SBMU.REC.1398.061). Also, this clinical study was registered with registration numbers 20181205041860N1 at 26 September 2019. All healthy donors and patients were informed about surgery and harvesting of BFP, preparation of BFP- tSVF, possible risks and complications, and then signed the informed consent.

\subsection{Experimental assessments}

\subsubsection{Harvesting and preparing of BFP}

The BFP tissues were obtained from three healthy male donors (age range of 25 -35 years) by a vestibular incision distal to the maxillary second molar. This tissue was exposed by blunt dissection while preserving the thin covering membrane (Figure 6A). Then approximately $4 \mathrm{ml}$ of the tissue was excised and delivered to the laboratory in Phosphate Buffered Saline (PBS) (Sigma-Aldrich, St. Louis, Missouri, the United States) supplemented with 5X penicillin/streptomycin antibiotic (Pen/Strep) ( Sigma-Aldrich, St. Louis, Missouri, the United States) and $1 \mathrm{X}$ amphotericin B (Sigma-Aldrich, St. Louis, Missouri, the United States). Also, cSVF and tSVF' isolation was performed according to the below protocols after dividing them to equal volume. 
1. Enzymatic (ENZ) protocol: The BFP tissue was washed with PBS (Sigma-Aldrich, St. Louis, Missouri, the United States) and incubated with an equal volume of type 1 collagenase enzyme $(3 \mathrm{mg} / \mathrm{ml})($ SigmaAldrich, St. Louis, Missouri, the United States) at $37^{\circ} \mathrm{C}$ for $30 \mathrm{~min}$ and shaker condition. After centrifugation, the resultant pellet was washed and cultured in the standard medium, containing Dulbecco's modified Eagle's medium (DMEM) (Thermo Fisher Scientific Waltham, MA, and the United States) supplemented with $15 \%$ fetal bovine serum (FBS) (Thermo Fisher Scientific Waltham, MA, and the United States), and $1 \mathrm{X}$ pen/strep antibiotic.

2. Mechanical protocol: Minceolip ${ }^{\circledR}$ device, was patented in the Intellectual Property Office of Real Estate Registration Organization of Iran (Application Number 13985040003006638) to isolation BFP- tSVF during surgery. This device manually disaggregated the minced BFP tissue into tSVF by passing the tissue from the filter (pore size is $100 \mu \mathrm{m}$ ) via a piston force (Figure $6(\mathrm{~B}-\mathrm{E})$ ). All parts of this device were fabricated from autoclaved materials (AISI 361L stainless steel and Fluorocarbon rubber), making it possible to sterilize by autoclave and use it several times. To perform experimental assessments, obtained BFP-tSVF were centrifuged at $500 \mathrm{~g}$ for 10 minutes. The supernatant was then thrown away, and the pellet was suspended and cultures in the standard medium.

\subsubsection{Cell counting, viability, and phenotypic analysis}

The number of nucleated cells obtained from BPF by the Minceolip ${ }^{\circledR}$ was freshly counted using a hemocytometer. According to the manufacturer, the percentage of BFP-tSVF viability was detected through Annexin V-FITC Apoptosis detected kit (Sigma-Aldrich, St. Louis, Missouri, and the United States)'s guideline. Briefly, the tSVF were washed with cold PBS twice and centrifuged at $300 \mathrm{~g}$ for 5 minutes. The pellet was mixed and incubated with $1 \mathrm{X}$ binding buffer, $2 \mu \mathrm{l}$ Propidium lodide (PI), and $1 \mu \mathrm{l}$ Annexin $\mathrm{V}$ for 10 minutes in darkness and room temperature.

Furthermore, to evaluate phenotypes, these BFP-tSVF were incubated in the blocking buffer and the various fluorochrome-conjugated antibodies for 30 minutes at $4^{\circ} \mathrm{C}$. These antibodies were CD90-FITC, CD44-FITC, CD31-PE, CD34-PE, CD45-PE, and CD14-FITC supplying from EXBio, Vestec, Czech Republic. The viability and phenotypic analysis were then carried out by flow cytometer (Applied Biosystems, Foster City, CA, USA) and FLOWJO software 7.6.1 (Tree Star Software, San Carlos, California, USA).।

\subsubsection{Cell culture and expansion rate}

The CSVF and tSVF were cultured in 25-T flasks under a humidified atmosphere containing $5 \%$ CO2 at $37^{\circ}$. The morphology of cells was microscopically seen to evaluate growth trends and possible infection. At confluence, $2 \times 10^{4}$ cells were detached using $0.25 \%$ trypsin/ 1 mM EDTA (Thermo Fisher Scientific Waltham, MA, and the United States) and seeded in the cell culture plate in the standard medium. The number of cells was counted at a density of approximately $70 \%$ using a hemocytometer up to eight passage numbers (PN). Then the expansion rate was calculated using the following formula (Equation 1) ${ }^{24}$; 


$$
\begin{gathered}
\text { Expansion rate }=\frac{\text { The number of cell at the end of culture }}{\text { The number of seed cells }} \\
\text { Equation 1. Expansion rate formula. }
\end{gathered}
$$

\subsubsection{Population doubling time}

The proliferation capability of isolated SVF using ENZ and mechanical protocols were assessed by calculating Population Doubling Time (PDT) based on the Patterson formula (Equation 2). To perform, a density of $2 \times 10^{4}$ of both SVF was cultured in the wells of 24 well plates with the standard medium. Then once every five days, the cells were detached using $0.25 \%$ trypsin/ $1 \mathrm{mM}$ EDTA and counted using a hemocytometer. Then the cells were re-cultured with an initial density.

Population doubling time $=\frac{\text { Duration }(\text { day }) \times \log (2)}{\log (\text { Final })-\log (\text { Initial })}$

Equation 2. Patterson formula for calculating population doubling time.

\subsubsection{Multi-lineage Differentiation potential}

Two types of cells with a density of $2 \times 10^{4}$ cells/well were seeded into 12 well plates and incubated in the standard medium at the one PN. After complete confluence, this medium was separately exchanged with Stem Pro (Life Technologies, California, United States), adipogenic medium (Life Technologies, California, Unite d States), and chondrogenic medium (Life Technologies, California, United States) to different into osteoblast, adipocyte and chondrocyte, respectively for 14 days. After the induction period, the cells were stained with Alizarin Red, Oil Red O, and Toluidine Blue to visualize calcium deposits, lipid droplets, and proteoglycans development, respectively, according to the manufacture's guidelines. The percentage of positive cells for all stains was quantified Image $\mathrm{J}$ (free download available at http://rsbweb.nih.gov/ij/) based on five images of each group.

\subsection{Clinical assessments}

\subsubsection{Design of the clinical study}

In the present study, the primary and secondary outcomes were to investigate the effectiveness, safety, and feasibility of isolated BFP- tSVF using the Minceolip ${ }^{\circledR}$ and the new bone formation after BFP- tSVF based therapy, respectively, in patients with maxillofacial bone defects. All the patients had the American society of anesthesiologists (ASA) I physical status classification system. According to eligibility criteria, ten patients with benign odontogenic tumors of the mandible were enrolled in this study, defined in Table 2. Our center's standard treatment procedure was to do a segmental or partial resection of the affected site and preserve the jaw with the reconstruction plate for at least one year. After that, the sites were reconstructed with the free non-vascular iliac bone graft. In this study, in half of the patients (BFP-tSVF group), the defects were filled with mechanical device derived BFP-tSVF with absorbable collagen sponge (AGS) (Gelfoam, Ethicon, Inc, Somerville, New Jersey) in the defect sites. Four patients underwent segmentally, and six patient's experienced 
partial resection of the tumor site (Table 3). The defect site was determined by a 3 D-printed surgical guide and cut without damage to blood vessels and inferior alveolar nerve.

\subsubsection{Safety and feasibility measurement}

Safety was considered an adverse event because there is not enough knowledge about the relationship between tSVF therapy and its complications in patients with bone defects. According to consolidated standards of reporting trials (CONSORT), the Adverse event is defined as any anticipated and unexpected harm after intervention according to consolidated standards of reporting trials (CONSORT) statement ${ }^{45}$. Hence the patients were carefully monitored for physical, radiological, and laboratory assessment by one expert oral-maxillofacial surgeon for one year after surgery, i.e., one week, one month, three months, and one year. The physical evaluation consisted of weight, body temperature, blood pressure, lymph node, neurological status. The radiological evaluation was screened by cone-beam computed tomography radiography. Laboratory assessments consisted of hematology index, C - reactive protein, erythrocyte sedimentation rate.

The protocols' feasibility was assessed according to five factors; surgical manipulation for BFP harvesting, sterilization of Minceolip device, convenience, time, and BFP-tSVF preparation cost.

\subsubsection{Radiographic assessment of the new bone formation}

Cone-beam computed tomography (CBCT) images were obtained to measure the bone density in the defect site twice, once before maxillofacial surgery (BD1) and once 12 months after it (BD2). The radiographic images were converted to 256 to 4096 grayscales and established the bone defect area using ImageJ software. Then, the percentage of bone density was calculated based on the grayscales ratio at BD2 to BD1 by the following formula (Equation 3);

$$
\text { Bone Density }(\%)=\frac{\text { The mean of the grayscales at } B D 2-\text { The mean of the grayscales at } B D 1}{\text { The mean of the grayscales at } B D 1} \times 100
$$

Equation 3. Bone Density formula.

This measure was performed thrice by one expert investigator, and the mean of the reported scales was used.

\subsection{Statistical analysis}

Statistical analyses, including unpaired two-tailed Student's t-test and two-way ANOVATukey's post-hoc, were carried out using GraphPad Prism Software (GraphPad Software, INC., La Jolla, Calif.). Quantitative data were obtained in triplicates and presented as mean \pm standard deviation (SD). P-value $<0.05$ was considered statistically significant.

\section{Declarations}

\section{Declarations}


The authors declare that there is no competing interest regarding the publication of this manuscript.

\section{Funding:}

The author(s) disclosed receipt of the following financial support for the research, authorship and/or publication of this article: This work is supported by the Iran Ministry of Health and Medical Education (Grant No: 700/1702) and the Department of Tissue Engineering and Applied Cell Sciences, Shahid Beheshti University of Medical Sciences, Tehran, Iran (Contact grant NO. 14647).

\section{Author Contributions:}

M.B and A.K convinced and designed the project and oversaw the collection of results. M.B, A.K and N.N helped the data analysis and discussion. A.K and F.B performed the operations. M.B, A.K, F.B and H.N wrote the manuscript.

\section{Acknowledgments:}

This study was supported by the Department of Tissue Engineering and Applied Cell Sciences, Shahid Beheshti University of Medical Sciences, Tehran, Iran, for providing technical support.

\section{References}

\section{Acknowledgments:}

This study was supported by the Department of Tissue Engineering and Applied Cell Sciences, Shahid Beheshti University of Medical Sciences, Tehran, Iran, for providing technical support.

\section{Funding:}

The author(s) disclosed receipt of the following financial support for the research, authorship and/or publication of this article: This work is supported by the Iran Ministry of Health and Medical Education (Grant No: 700/1702) and the Department of Tissue Engineering and Applied Cell Sciences, Shahid Beheshti University of Medical Sciences, Tehran, Iran (Contact grant NO. 14647).

\section{Author Contributions:}

M.B and A.K convinced and designed the project and oversaw the collection of results. M.B, A.K and N.N helped the data analysis and discussion. A.K and F.B performed the operations. M.B, A.K, F.B and H.N wrote the manuscript.

\section{Competing Interests:}

The authors declare that there is no competing interest regarding the publication of this manuscript. 


\section{Tables}

Table 1. Population doubling time (days) of isolated cells through ENZ and mechanical protocol.

\begin{tabular}{c|cc} 
Time & ENZ & Mechanical \\
\hline $\mathbf{5}$ & $1.46 \pm 0.74$ & $2.06 \pm 0.69$ \\
$\mathbf{1 0}$ & $2.01 \pm 0.78$ & $1.92 \pm 0.11$ \\
$\mathbf{1 5}$ & $2.61 \pm 0.16$ & $2.56 \pm 0.11$ \\
$\mathbf{2 0}$ & $2.31 \pm 0.22$ & $2.19 \pm 0.10$ \\
$\mathbf{2 5}$ & $3.00 \pm 0.40$ & $2.55 \pm 0.52$ \\
$\mathbf{3 0}$ & $8.16 \pm 1.25^{*}$ & $5.02 \pm 1.08^{*}$ \\
$\mathbf{3 5}$ & $15.05 \pm 0.58^{\#}$ & $8.9 \pm 0.48^{\#}$ \\
\hline
\end{tabular}

ENZ: Enzymatic protocol. *\# p Value $<0.0001$

Table 2. Patient eligibility criteria

\begin{tabular}{ll} 
Inclusion criteria & Exclusion criteria \\
\hline Having maxillofacial defect & Immune system disorders \\
\hline$>18$ years age & Uncontrolled diabetes \\
\hline Non-smokers & Ongoing chemotherapy or radiation \\
\hline Signed informed consent & Drug abuse \\
\hline & Chronic sinus pathology \\
\hline & Pregnancy or lactation \\
\hline & Active infection
\end{tabular}

Table 3. Demographic and clinical information of patients. 


\begin{tabular}{|c|c|c|c|c|c|c|c|c|}
\hline \multicolumn{2}{|l|}{ Patient } & \multicolumn{2}{|c|}{ Age } & Gender $\begin{array}{c}\text { Final } \\
\text { diagnosis }\end{array}$ & pre & $\begin{array}{l}\text { Cell } \\
\text { product }\end{array}$ & $\begin{array}{c}\text { Cell } \\
\text { carries }\end{array}$ & $\begin{array}{l}\text { Adverse } \\
\text { events }\end{array}$ \\
\hline \multicolumn{9}{|c|}{ BFP-tSVF group } \\
\hline 1 & \multirow[t]{2}{*}{$\begin{array}{c}\text { Segmental } \\
\text { resection }\end{array}$} & 49 & Male & $\begin{array}{c}\text { Bilateral central } \\
\text { giant cell } \\
\text { granuloma }\end{array}$ & $\begin{array}{c}\text { Right } \\
\text { and left, } \\
\text { mandible }\end{array}$ & $\begin{array}{l}\text { BFP- } \\
\text { Tsvf }\end{array}$ & AGS & - \\
\hline 2 & & 32 & Male & Ameloblastoma & $\begin{array}{c}\text { left, } \\
\text { maxilla }\end{array}$ & $\begin{array}{l}\text { BFP- } \\
\text { tSVF }\end{array}$ & AGS & - \\
\hline 3 & \multirow[t]{3}{*}{$\begin{array}{l}\text { Partial } \\
\text { resection }\end{array}$} & 33 & Female & Ameloblastoma & $\begin{array}{c}\text { Left, } \\
\text { mandible }\end{array}$ & $\begin{array}{l}\text { BFP- } \\
\text { tSVF }\end{array}$ & AGS & $\begin{array}{c}\text { Oral } \\
\text { inflammatory } \\
\text { exudates and } \\
\text { pain after } \\
\text { eight months }\end{array}$ \\
\hline 4 & & 27 & Male & Ameloblastoma & $\begin{array}{c}\text { Left, } \\
\text { mandible }\end{array}$ & $\begin{array}{l}\text { BFP- } \\
\text { tSVF }\end{array}$ & AGS & - \\
\hline 5 & & 18 & Female & Cystic lesions & $\begin{array}{l}\text { Central, } \\
\text { mandible }\end{array}$ & $\begin{array}{l}\text { BFP- } \\
\text { tSVF }\end{array}$ & AGS & - \\
\hline \multicolumn{9}{|c|}{ Control group } \\
\hline 6 & \multirow[t]{2}{*}{$\begin{array}{c}\text { Segmental } \\
\text { resection }\end{array}$} & 19 & Female & Ameloblastoma & $\begin{array}{c}\text { Right, } \\
\text { mandible }\end{array}$ & - & - & - \\
\hline 7 & & & Male & Ameloblastoma & $\begin{array}{c}\text { Left, } \\
\text { mandible }\end{array}$ & - & - & - \\
\hline 8 & \multirow[t]{3}{*}{$\begin{array}{c}\text { Partial } \\
\text { resection }\end{array}$} & 34 & Female & Ameloblastoma & $\begin{array}{c}\text { Right, } \\
\text { mandible }\end{array}$ & - & - & - \\
\hline 9 & & 45 & Male & Ameloblastoma & $\begin{array}{c}\text { Left, } \\
\text { mandible }\end{array}$ & - & - & - \\
\hline 10 & & 61 & Male & Cystic lesions & $\begin{array}{l}\text { Central, } \\
\text { mandible }\end{array}$ & - & - & - \\
\hline
\end{tabular}

BFP: Buccal Fat Pad, tSVF: Tissue Stromal Vascular Fraction, AGS: Absorbable Collagen Sponge

\section{Figures}



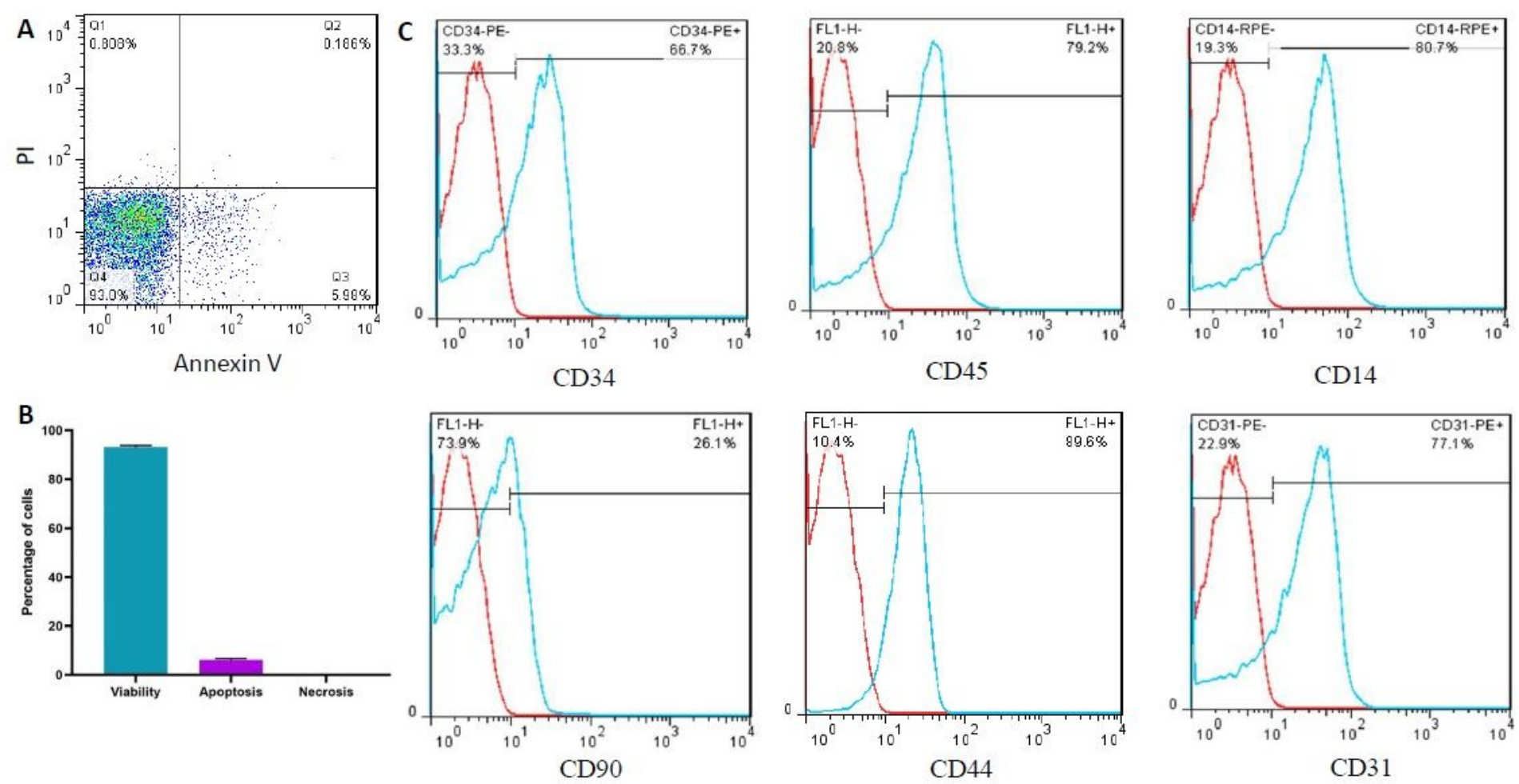

\section{Figure 1}

Flow cytometer analysis of freshly BFP-tSVF. A) Dot plot shows viable and apoptotic BFP-tSVF by Annexin V$\mathrm{PI}$ staining after isolation using the Minceolip ${ }^{\circledR}$ device. $\left.\mathrm{B}\right)$ The bar graph demonstrates the average of the percentage of viable and apoptotic BFP-tSVF. C) Histograms show fluorescence intensity and percentage expression of surface markers of BFP-tSVF. BFP: Buccal Fat Pad, tSVF: tissue Stromal Vascular Fraction, PI: Propidium lodide. The results are presented as mean \pm SD from three healthy donors. 

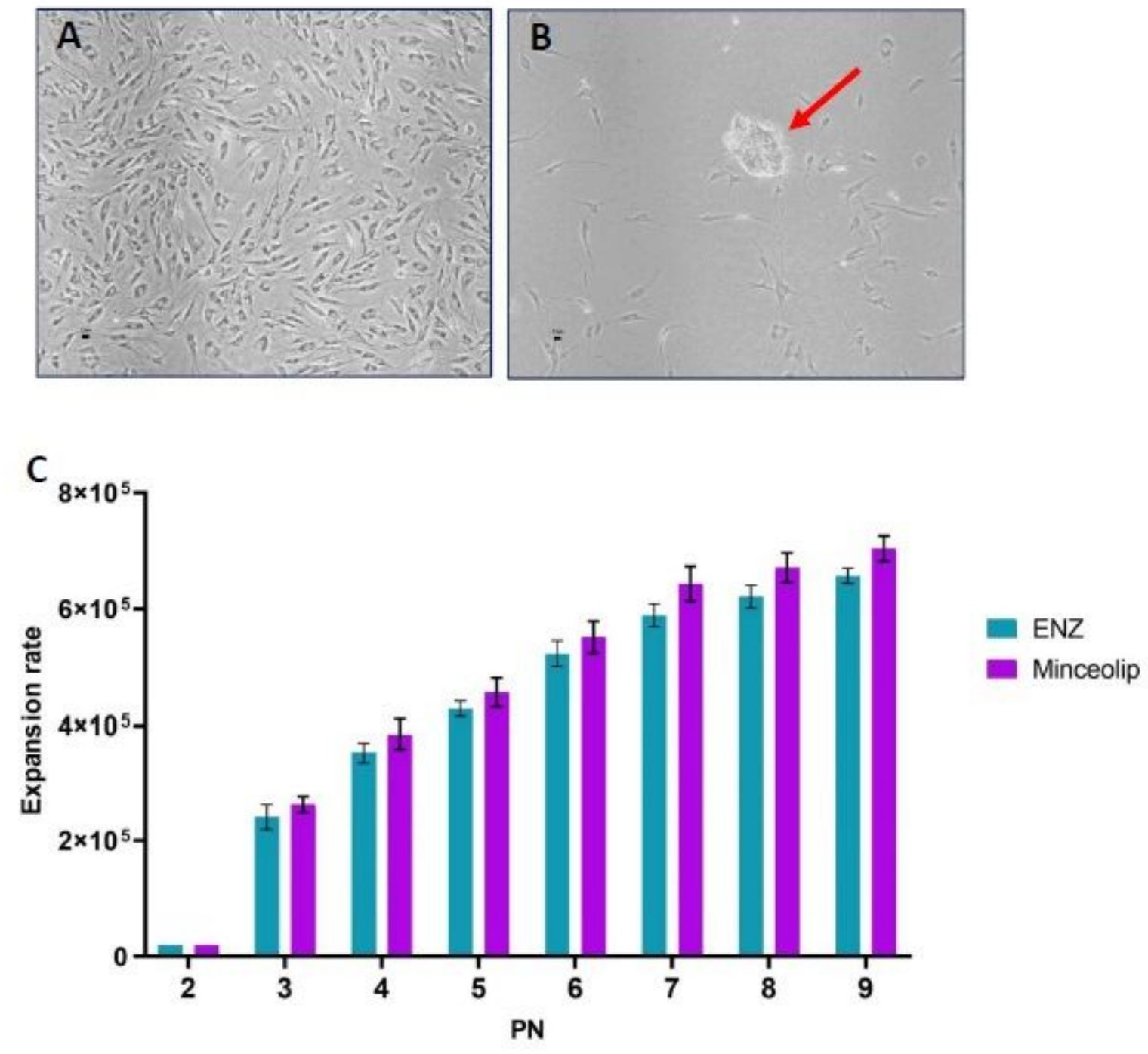

Figure 2

Experimental assessment of BFP-tSVF. A) Morphology of fibroblast-like cells was isolated using ENZ protocol after seven days from in vitro culture. B) Morphology of fibroblast-like cells and tSVF were isolated using Minceolip ${ }^{\circledR}$ protocol and tSVF fragment (red arrow). C) Evaluation of the expansion rate of isolated cells using ENZ and Minceolip device for nine passages. ENZ: Enzyme, BFP: Buccal Fat Pad, tSVF: Tissue Stromal Vascular Fraction. Scale bar $=12 \mu \mathrm{m}$. The results are presented as mean \pm SD from three healthy donors. 
A Osteogenesis
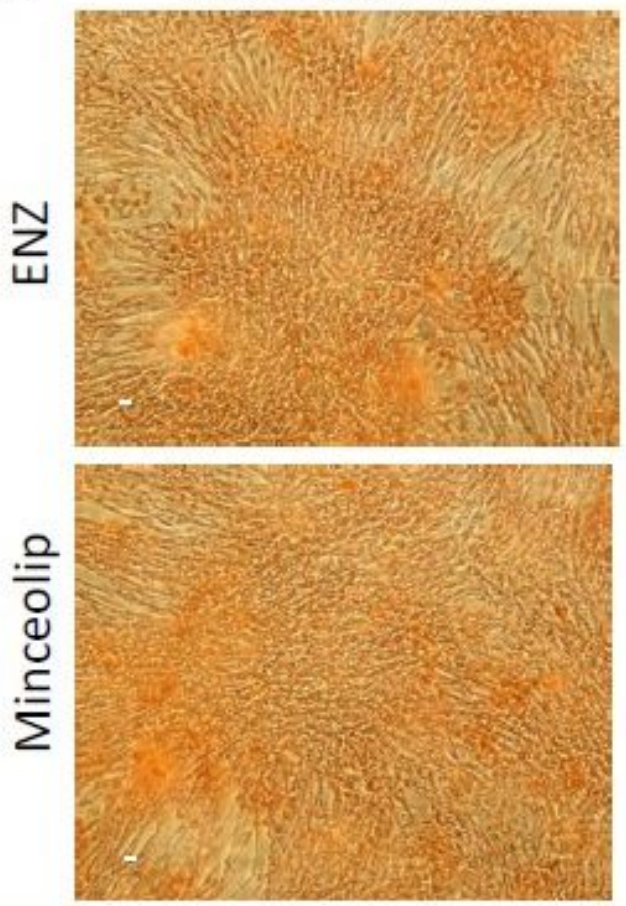

B

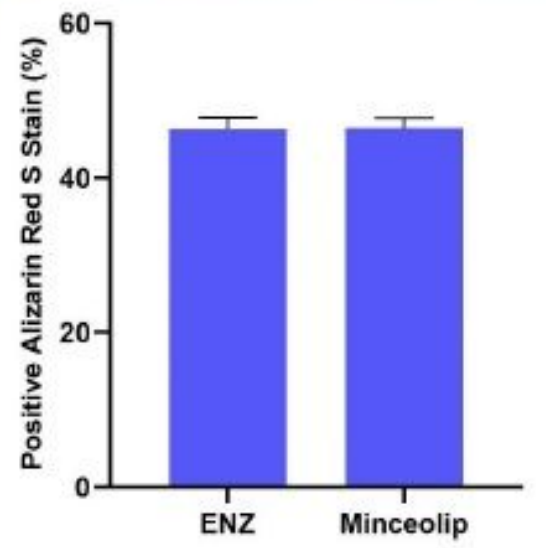

Adipogenesis
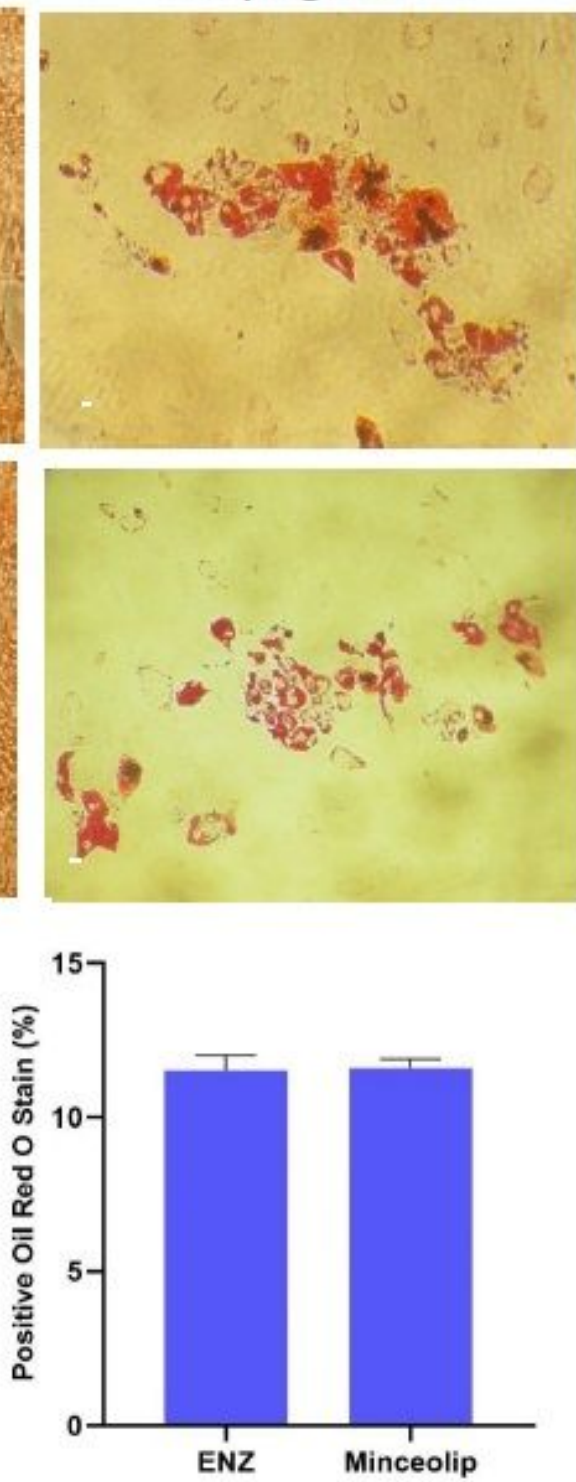

Chondrogenesis
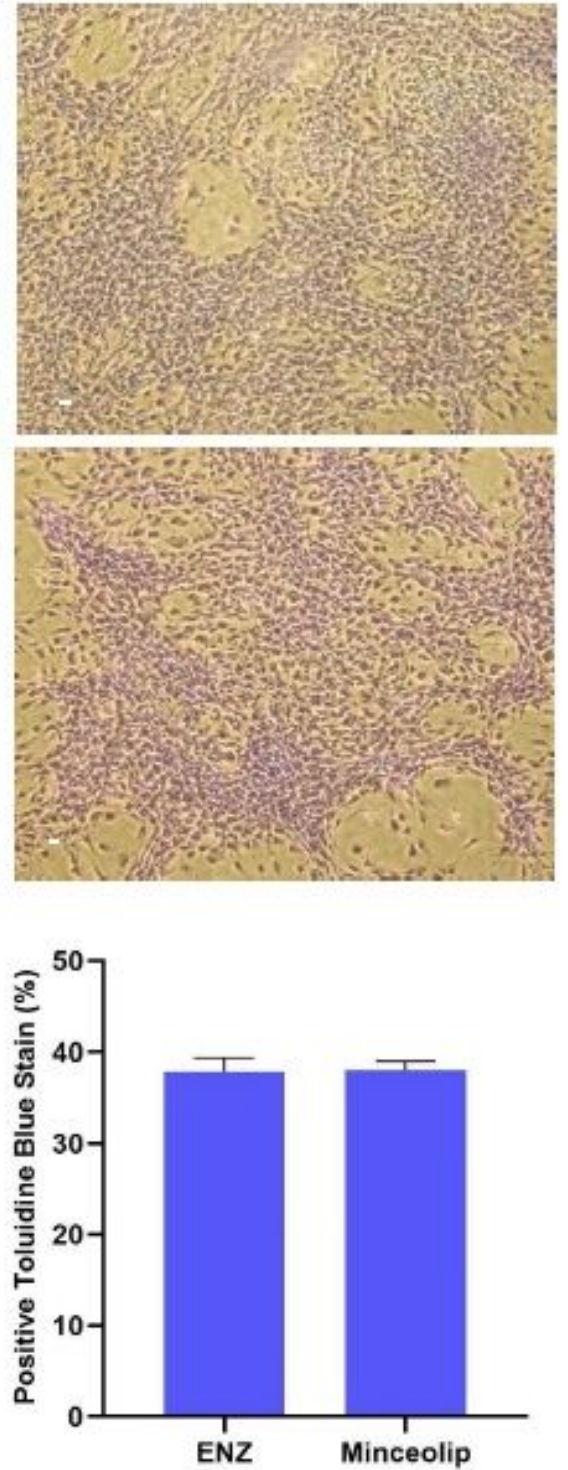

\section{Figure 3}

Multi-lineage differentiation of cells isolated using ENZ and mechanical protocols. A) Specific staining, including Alizarin red, Oil Red O, and Toluidin Blue, for confirmation of osteogenesis, adipogenesis, and chondrogenesis. B) Assessment of The percentage of positive cells for these specific staining. Error bars are $\mathrm{SD}(\mathrm{N}=3)$. ENZ: Enzyme. The results are presented as mean $\pm \mathrm{SD}$ from three healthy donors-the scale bar $=12$ $\mu \mathrm{m}$. 

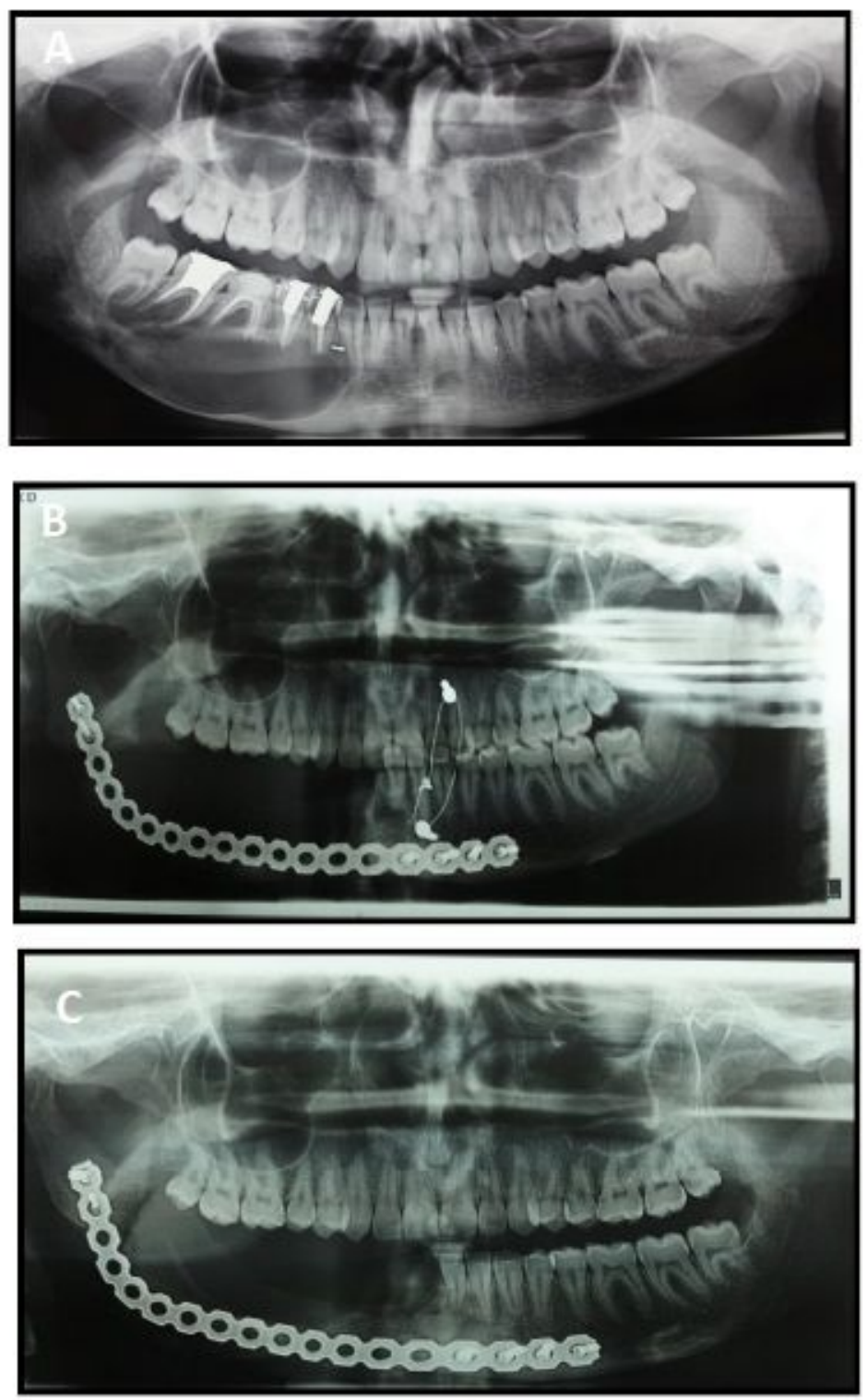

\section{Figure 4}

Radiography image from a benign tumor in a patient who was treated using standard segmental resection surgical procedure. A) Tumor site before surgery, B) image after surgery to remove the tumor, and C) image of defect site after one year. 

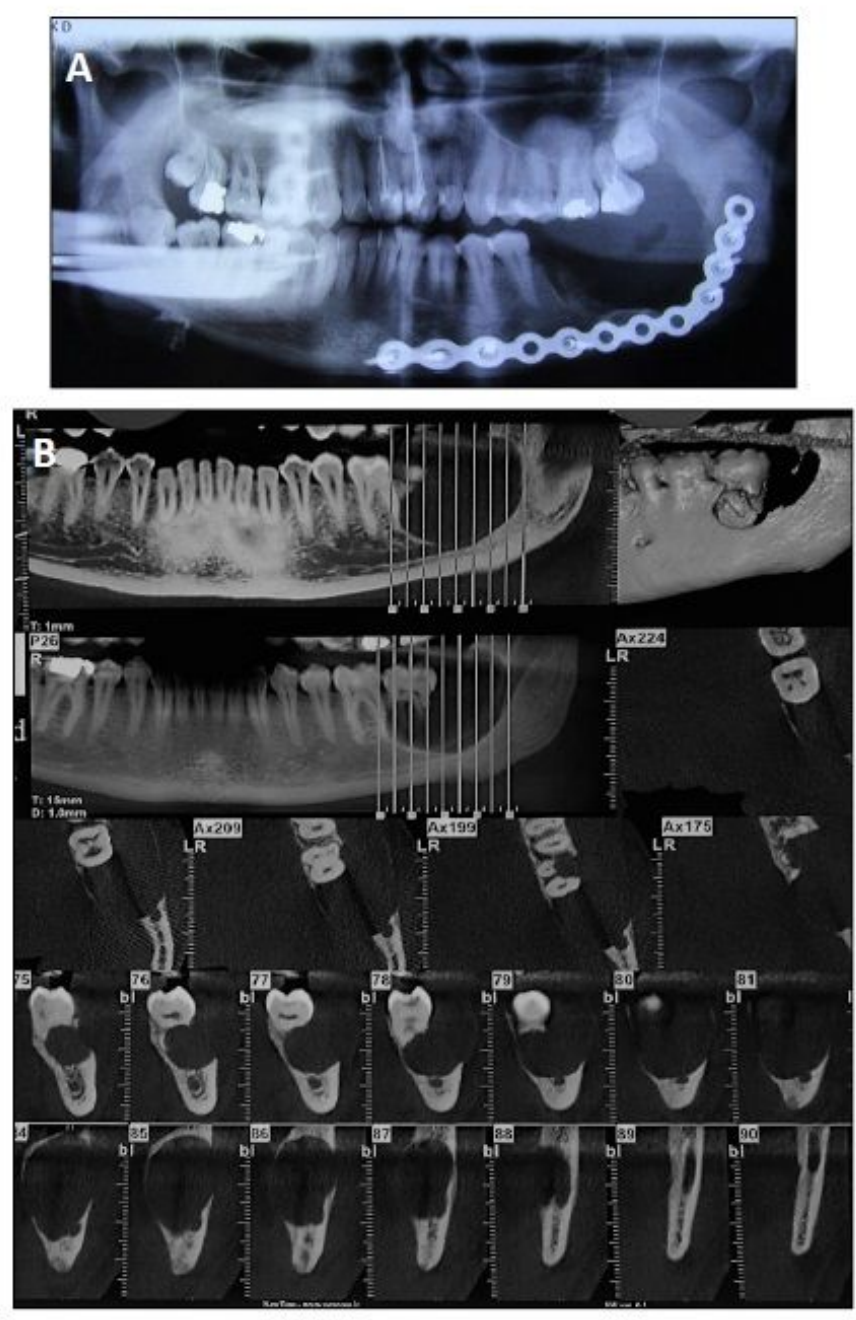
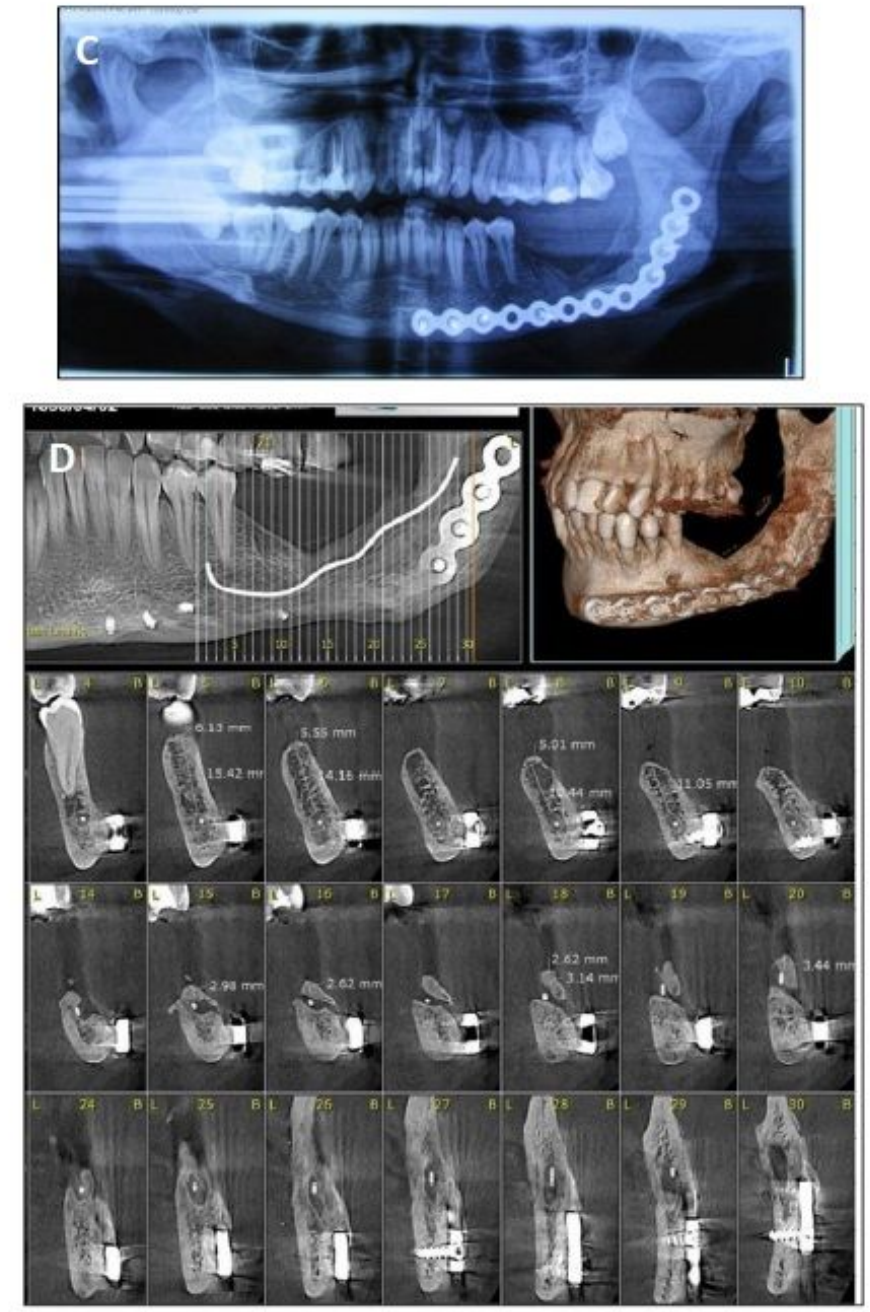

\section{Figure 5}

The radiographic evaluation in a patient who received BFP-tSVF using partial resection technique on the left side mandible. A and B) OPG and CBCT Before surgery, respectively. C and D) OPG and CBCT after one year, respectively. 

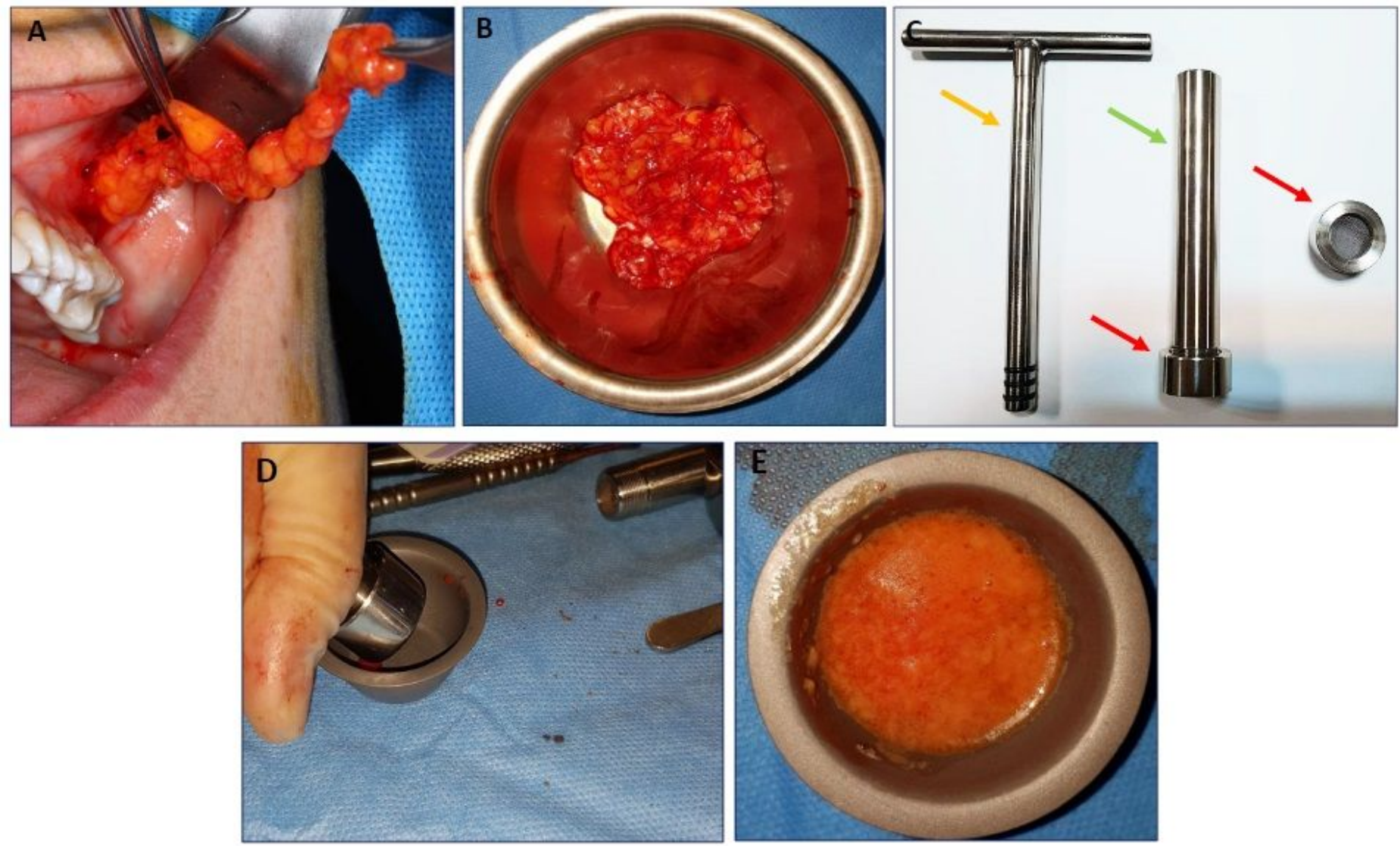

\section{Figure 6}

Processes of isolated BFP-tSVF. A) BFP harvesting during maxillofacial surgery. B) Transferring the tissue into a sterile gallipot. C) Menceolip device, including piston (orange arrow), tissue chamber (green arrows), and filter (red arrows). D) Passing the BFP tissue from the filter by the piston. E) BFP-tSVf. BFP: Buccal Fat Pad, tSVF: tissue Stromal Vascular Fraction. 